\title{
Epidemiological analysis of a methicillin-resistant Staphylococcus aureus outbreak using restriction fragment length polymorphisms of genomic DNA
}

\author{
Wafa El-Adhami, ${ }^{1}$ Leslee Roberts, ${ }^{2}$ Alison Vickery, ${ }^{3}$ Barbara Inglis, ${ }^{1}$ Adrian Gibbs ${ }^{4}$ \\ and Peter R. StewarT ${ }^{1 *}$ \\ ${ }^{1}$ Division of Biochemistry and Molecular Biology, Faculty of Science, Australian National University, \\ Canberra ACT 2601, Australia \\ ${ }^{2}$ Department of Microbiology, Woden Valley Hospital, Canberra ACT 2605, Australia \\ ${ }^{3}$ Department of Microbiology, Royal Prince Alfred Hospital, Camperdown NSW 2050, Australia \\ ${ }^{4}$ Research School of Biological Sciences, Australian National University, Canberra ACT 2601, Australia
}

(Received 12 April 1991; revised 11 July 1991; accepted 28 August 1991)

\begin{abstract}
The genomic DNA of 58 isolates of methicillin-resistant Staphylococcus aureus (MRSA) obtained during an infection outbreak at two major Canberra hospitals was analysed for restriction fragment length polymorphism (RFLP) by digestion with the endonuclease SmaI and resolution of the fragments by pulsed-field gel electrophoresis. Based on the fraction of common fragments generated by the endonuclease, DNA similarities among the isolates were estimated. Distance matrix analysis showed that the MRSA isolates could be divided into two major clusters (RFLP types I and II) and one minor one (type 46). A fourth group of miscellaneous isolates was found to be heterogeneous in terms of DNA sequence similarity. The epidemiological data indicated that RFLP type I was most common in the intensive care units in the two hospitals, with particular subtypes of RFLP type I concentrated in individual units. RFLP type II and the miscellaneous group were more generally distributed. Type 46 isolates appear to be related to a group which was present in epidemics in Melbourne hospitals in the early 1980s. Using the standard phage set, the RFLP type I group was largely untypable. However, type II isolates were all phage typable, with a shared susceptibility to phages 29/85/95/90; type 46 isolates had a shared susceptibility to phages $85 / 90$. The miscellaneous isolates were of variable phage types.
\end{abstract}

\section{Introduction}

Infections due to methicillin-resistant Staphylococcus aureus (MRSA) often reach epidemic proportions in hospitals throughout the world. Accurate identification of MRSA strains or types, and their carriers within a hospital, are of special importance in coping with these outbreaks, and a range of methods designed to do this have been described (Berke \& Tilton, 1986; Coia et al., 1990; Cookson et al., 1986; Mulligan et al., 1988; Skurray et al., 1988; Smith \& Berezny, 1986; Vickery et al., 1986).

We have sought a method which would provide a measure of genetic similarities between staphylococcal isolates, and thus permit the more precise genetic specification of clones or strains causing outbreaks. This

\footnotetext{
Abbreviations: FIGE, field inversion gel electrophoresis; MRSA, methicillin-resistant Staphylococcus aureus; RFLP, restriction fragment length polymorphism.
}

could eventually lead to the measuring of evolutionary change in strains or species within the hospital environment as a function of time, and from this an early warning of particularly troublesome strains.

One approach to this is by measuring sequence similarity through hybridization analysis of total genomic DNA from a range of MRSA isolates. While technically straightforward this method lacks the necessary discrimination, since within the genus Staphylococcus intraspecies variation in bulk DNA hybridization appears to be $5 \%$ or less (Schleifer \& Fischer, 1982). Strains or clones within a species may be too closely related to permit reliable discrimination by hybridization of total genomic DNA from different isolates.

A more sensitive method for detecting sequence dissimilarities is by analysis of differences in size and number of fragments generated by restriction endonuclease digestion of DNA, that is, by the quantitative analysis of restriction fragment length polymorphisms 
(RFLPs). In this case, the DNA molecule is 'sampled' for sequences that are cleaved by a particular restriction endonuclease; fragments of the same length and number are generated from genomes of identical or very similar sequence, but of different length and number where the sequences are different. Quantitatively, the degree of sequence difference can be estimated by comparing multiple fragments after digestion of intact, large (genomic) tracts of DNA with an endonuclease which cleaves at a sufficient, but not excessive, number of sites. The proportion of fragments which have identical sizes, measured by electrophoresis, provides an estimate of the sequence similarity of the two DNA molecules. These patterns, provided they are not excessively complex (i.e. the number of fragments generated by the endonuclease permits a precise identification of the relative mobility of each fragment) can then be subjected to numerical analysis to give statistical estimates of sequence similarity (Upholt, 1977; Nei \& Li, 1979).

We have used this approach to examine isolates of $S$. aureus collected during an MRSA epidemic in the two major public hospitals in Canberra. We used the restriction endonuclease SmaI to digest cellular DNA after lysis of cells in situ in agarose gels, and field inversion gel electrophoresis (FIGE; Carle et al., 1986) to separate the fragments generated by this endonuclease (Pattee, 1990). Numerical analysis of the fragment patterns was used to generate a sequence similarity matrix, and from this the genetic relatedness of the isolates was calculated. This was then correlated with epidemiological data and to phage typing of the isolates.

\section{Methods}

Bacterial isolates. MRSA jsolates were obtained from patients (only one isolate from each patient) in the Royal Canberra and Woden Valley Hospitals (Australian Capital Territory) during May-September 1989. Few MRSA isolates are normally found in these hospitals, but in this particular period, 58 isolations were recorded. Isolates were identified as $S$. aureus by growth on mannitol/salt agar, and by positive coagulase and DNAase tests. Antibiotic susceptibilities were determined by a standard agar dilution method (National Committee for Clinical Laboratory Standards, 1986). Resistance to the heavy metal ions cadmium and mercury was determined as described by Lyon et al. (1983). Phage typing of the isolates was done according to the method of Blair \& Williams (1961).

Preparation and digestion of DNA for FIGE. Cells were embedded in agarose, cell walls digested, and DNA cut with endonucleases as described by Smith et al. (1986) and modified for $S$. aureus by Inglis $e t$ al. (1990). FIGE was done as described by Carle et al. (1986) and modified by Inglis et al. (1990). Molecular sizes of DNA fragments were determined by comparison with $\lambda$ phage concatemers and the genomic fragments from $S$. aureus strain ANS46 (Inglis et al., 1990).

Estimation of nucleotide sequence diversity between MRSA isolates. The method of Nei \& $\mathrm{Li}$ (1979) and Upholt (1977) was used to calculate the degree of sequence similarity of cellular DNA in the MRSA isolates. Numbers and mobilities of fragments were compared on Polaroid photographs of FIGE gels stained with ethidium bromide. $F$, the proportion of shared fragments in the two genomes (also known as the coefficient of similarity), was calculated as

$$
F=2 n_{\mathrm{xy}} /\left(n_{\mathrm{x}}+n_{\mathrm{y}}\right)
$$

where $n_{\mathrm{x}}$ is the total number of DNA fragments from strain $\mathrm{X}, n_{\mathrm{y}}$ the total number from strain $\mathrm{Y}$, and $n_{\mathrm{xy}}$ the number of fragments identical in the two strains (Nei \& $\mathrm{Li}, 1979$ ). $F$ was then used to determine $p$, the estimated fraction of nucleotides different for the two DNA samples, from the relationship

$$
p=1-\frac{\left[\left(F^{2}+8 F\right)^{1 / 2}-F\right]^{1 / n}}{2}
$$

derived by Upholt (1977), where $n$ is the number of base pairs recognized by the endonuclease, and has a value of 6 for SmaI. A matrix of values of $p$ for all pairs of isolates was constructed, and a dendrogram computed using the neighbour-joining method of Saitou \& Nei (1987).

\section{Results}

\section{Antibiotic resistance phenotypes of MRSA isolates}

All 58 isolates examined were resistant to penicillin, methicillin, erythromycin and clindamycin; 56 were resistant to cotrimoxazole, tetracycline and trimethoprim; and 53 were resistant to gentamicin. Twelve isolates were resistant to chloramphenicol, and two to fusidic acid; none were resistant to vancomycin or rifampicin. Resistance to cadmium and mercury was seen in 55 and 45 isolates respectively.

\section{Source of isolates}

The 58 isolates examined were from: (1) neonates born at the Royal Canberra ( $\mathrm{RCH})$ or Woden Valley Hospitals (WVH) and transferred to the RCH neonatal intensive care nursery; and nurses working in this nursery with no history of MRSA $(n=22)$; (2) patients in the $\mathrm{RCH}$ intensive care unit who had not been patients in another hospital within the last 6 months and had not had MRSA colonization or infection $(n=13)$; (3) patients already colonized with MRSA when admitted to the Canberra hospitals, or who had acquired MRSA elsewhere $(n=8)$; (4) patients whose sources of infection were not in categories $1-3(n=15)$.

\section{FIGE analysis following SmaI digestion of cellular DNA}

Electrophoresis of SmaI digests yielded well-resolved patterns of 19-22 fragments of $10-700 \mathrm{~kb}$ in 57 isolates tested; one further isolate yielded only 13 fragments. We did not test independently for the presence of plasmids in the isolates. Plasmids greater in size than $10 \mathrm{~kb}$ and containing one or no SmaI sites would have been detected as a single fragment on these FIGE gels, and estimates of genetic difference affected accordingly. 


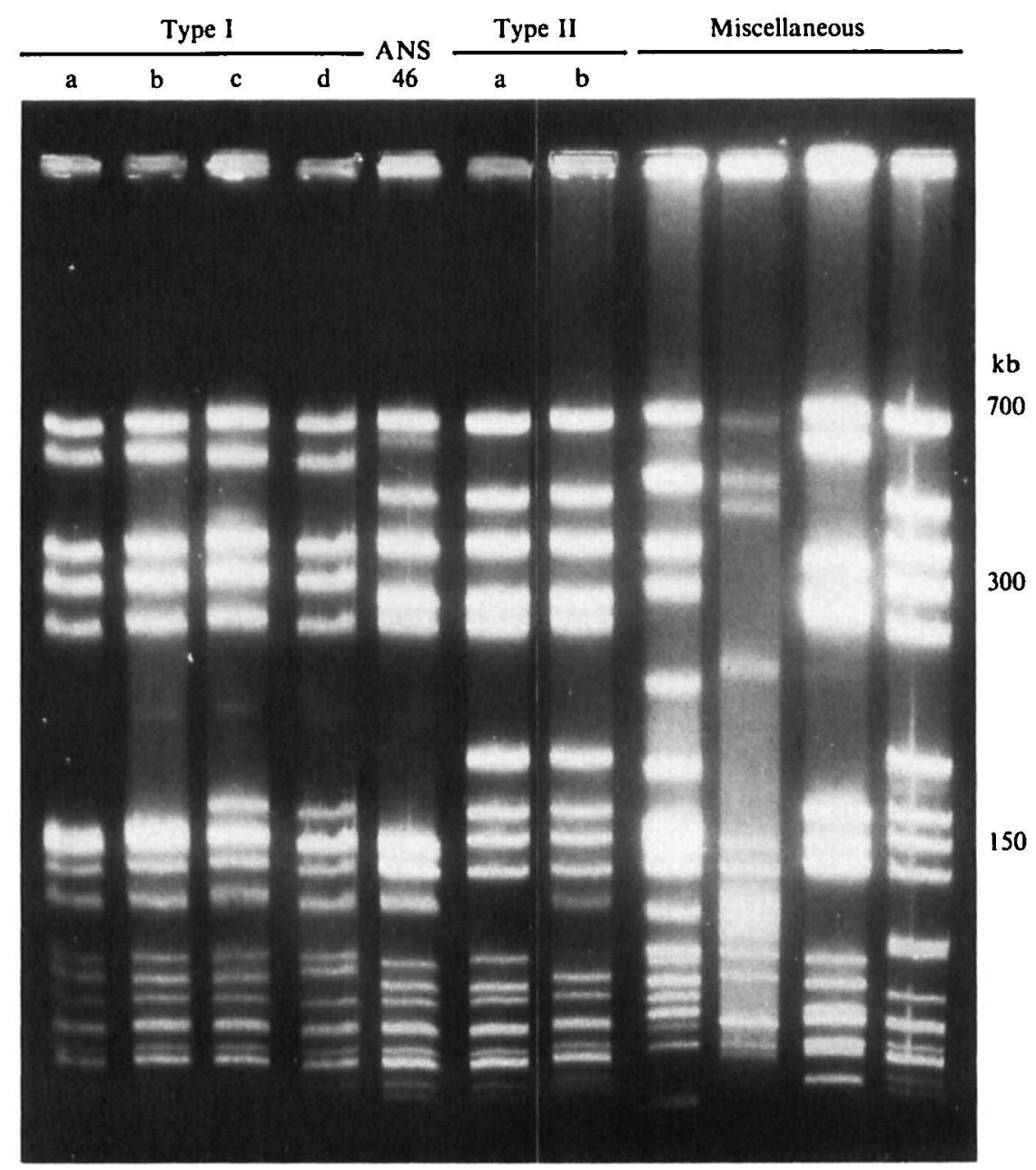

Fig. 1. FIGE of genomic DNA from RFLP types I, II and 46, and the miscellaneous group. Genomic DNA was digested with SmaI and FIGE done as described in Methods. A fragment size scale is shown on the right.

As the $S$. aureus genome is approximately $3 \times 10^{6} \mathrm{bp}$ and contains $35 \mathrm{~mol} \% \mathrm{G}+\mathrm{C}$ (Pattee, 1990), SmaI (recognition site 5'-CCCGGG-3') should cleave the intact genome into 80-90 fragments if it were a randomized sequence. Since fewer than one-quarter of this number is seen, either the Smal recognition sequence is under-represented in the $S$. aureus chromosome, or many small fragments are generated by this endonuclease. FIGE analyses designed to detect fragments as small as $3 \mathrm{~kb}$ indicate that the latter is unlikely to be the case.

The gel banding patterns of the isolates fell into two major clusters (referred to as types I and II), plus a miscellaneous group which shared fewer common fragments. A minor fourth group of three isolates (type 46) contained strain ANS46, which is an MRSA originally isolated during an epidemic in Melbourne in 1982 and which has been extensively studied in this laboratory including analysis by FIGE (Heneine \& Stewart, 1986; Matthews et al., 1987; Inglis et al., 1990). Fig. 1 shows representative FIGE patterns for members of the four different types.

Types I and II divided into further subgroups according to smaller differences in the pattern of SmaI fragments. Fig. 2 compares schematically the SmaI fragments found in types I, II and 46 and their subtypes. Of the total isolates, $63 \%$ were type I, $21 \%$ were type II, $4 \%$ were type 46 , and $12 \%$ were in the miscellaneous group.

Restriction endonuclease fragment patterns were stable features of the isolates and of the DNA isolation procedure. When the isolates were repeatedly subcultured, their DNA yielded unchanged SmaI fragment patterns. 


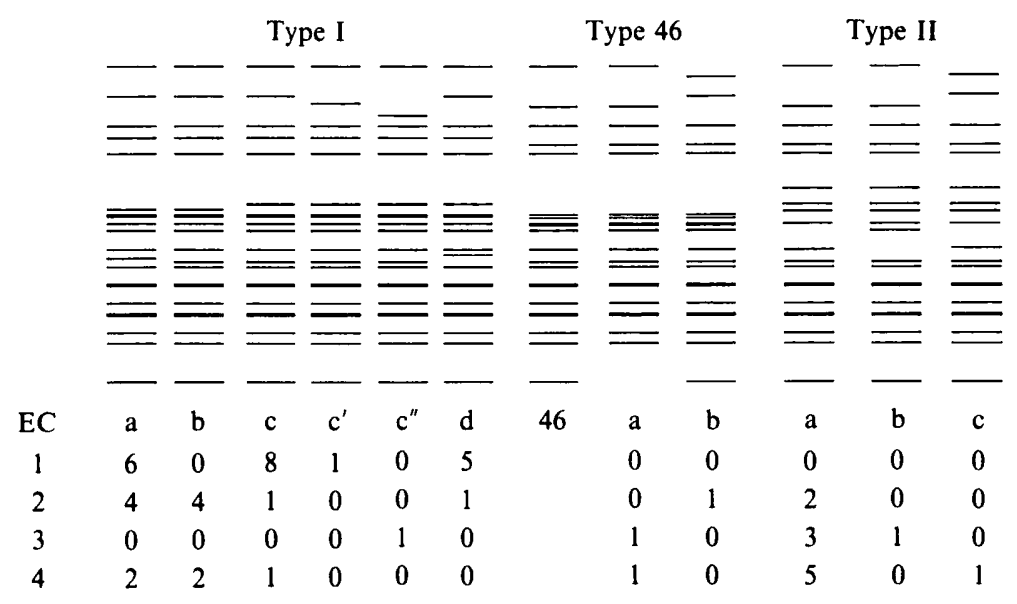

Fig. 2. Schematic summary of fragments of DNA from RFLP types I, II, and 46 obtained after FIGE of SmaI digested genomic DNA. Subtypes are illustrated, and the distribution of these is shown (numbers of strains) among the epidemiological categories (EC).

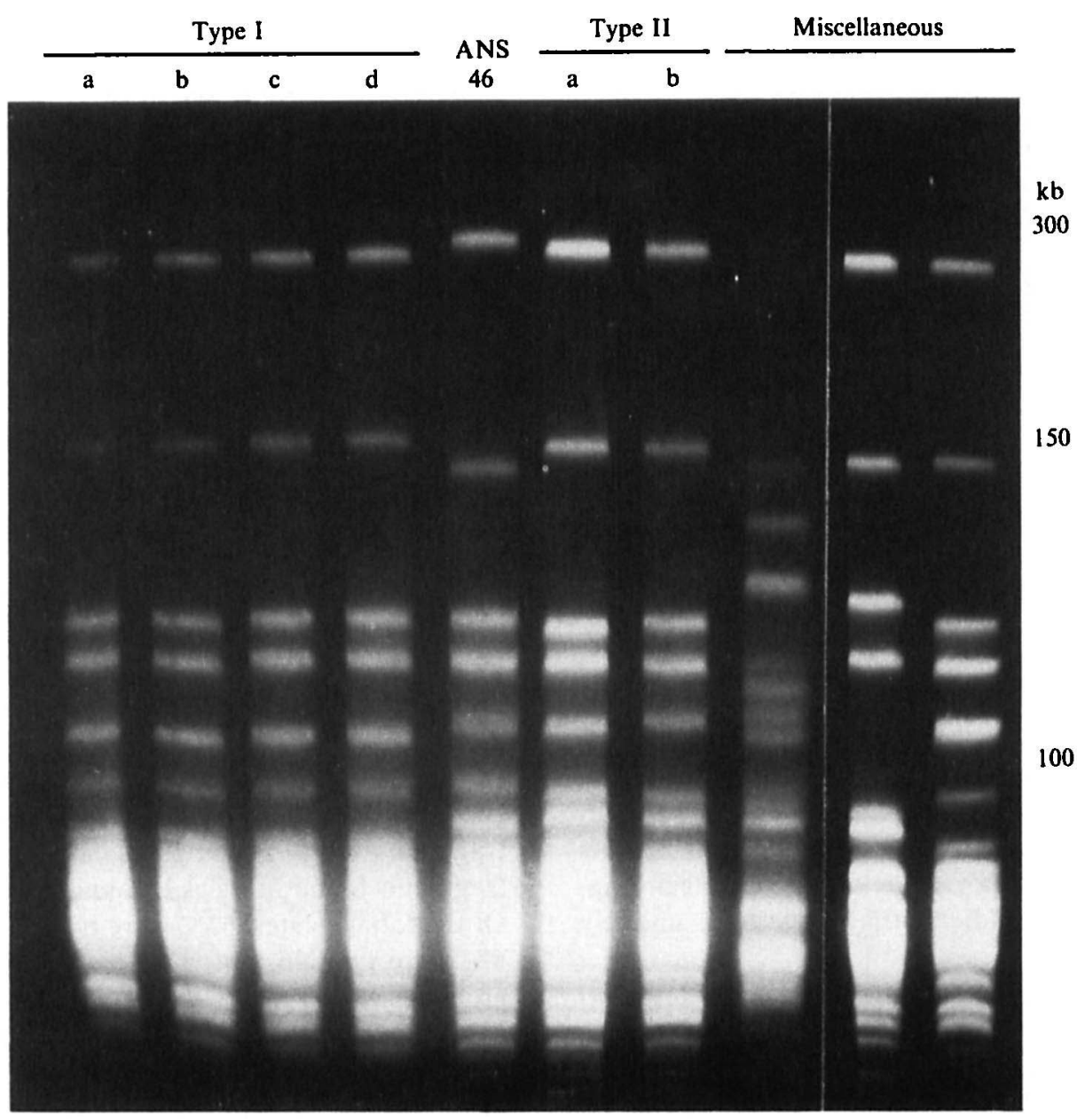

Fig. 3. Fragments obtained by FIGE of genomic DNA digested with BssHII. A fragment size scale is shown on the right. 
FIGE analysis of cellular DNA digested with other endonucleases

The group of MRSA isolates into major types, established using $S m a I$, was confirmed using another rarecutting endonuclease, BssHII, to generate fragment patterns. BssHII recognizes the sequence 5'-GCGCGC-3', and would be expected to generate the same number of fragments as $S m a$ I from a randomized sequence the size and composition of the $S$. aureus genome. However, as Fig. 3 shows, the number observed is substantially larger than that seen with SmaI; 30-40 bands larger than $10 \mathrm{~kb}$ can be seen, and a further 10 or so smaller ones are lost from the gel. Though the crowding of fragments does not permit the accurate counting of bands and comparison of their sizes, the overall gel patterns bear out the general divisions into types distinguished by SmaI. The experiments with BssHII show that an optimum number of bands for comparative purposes is about 20 ; enzymes generating more provide less usable quantitative data.

SacII (5'-CCGCGG-3'), like $S m a I$, also generated 2030 fragments from $S$. aureus DNA, but for some reason bands on gels from SacII digests did not show the sharpness seen with SmaI. This enzyme was therefore not used further. EcoRI, HindIII and BglII cleave $S$. aureus DNA too frequently to be useful in the present analysis.

\section{Size of the MRSA chromosome}

Summing the sizes of individual fragments generated by SmaI gives an estimate of the total genome size of these MRSA isolates. For five different subtypes the mean size ( \pm range) was $3106 \pm 118 \mathrm{~kb}$.

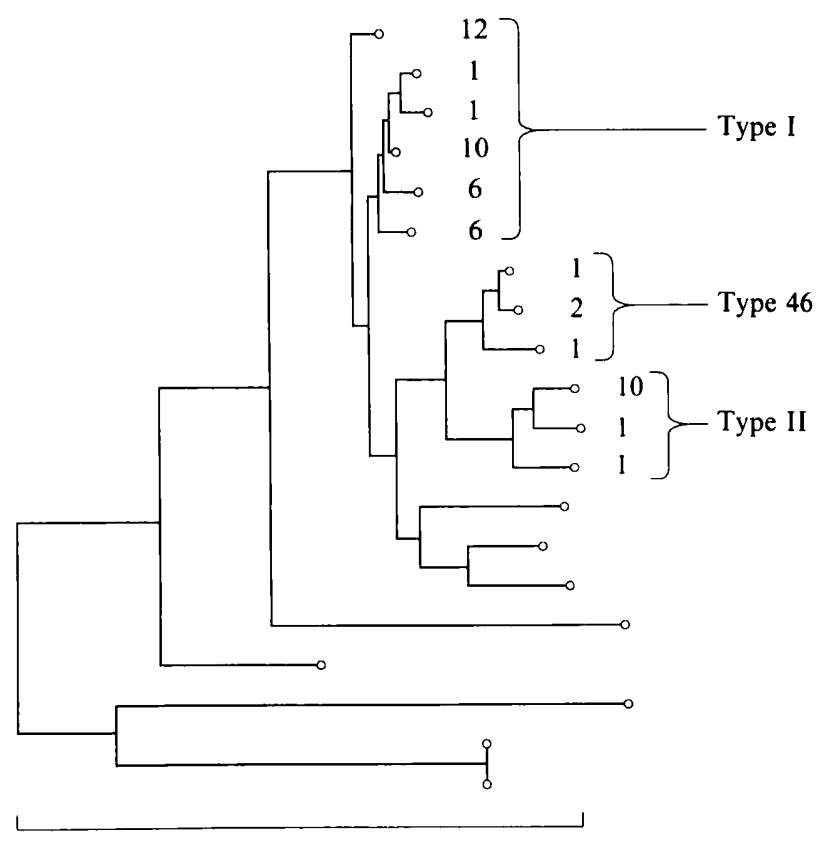

Fig. 4. Dendrogram calculated from the $p$ values for genomic DNA from all isolates. Open circles represent subtypes, and the numerals indicate the number of isolates of each subtype that were found. Types I, II, and 46 are shown; the remainder belong to the miscellaneous group. The genetic distance $(p)$ is represented by the horizontal distances in the dendrogram connecting isolates; the bar indicates a $p$ value of 0.04 . The methods for calculating and constructing the dendrogram are described in Methods.

Genetic relationships determined by numerical analysis of fragment length polymorphisms

Values of $F$ (the fraction of common fragments) were calculated as described in Methods, and from these,

Table 1. Distribution of MRSA isolates among RFLP types, epidemiological categories, and phage types

Epidemiological categories are as described in the text: (1) isolates from a neonatal intensive care nursery, (2) isolates from an intensive care unit, (3) isolates from sources outside Canberra, and (4) isolates of unknown origin. Figures across the table indicate the number of isolates of a particular phage type, and the figures in parentheses refer to the phage types to which the isolates are susceptible. (nt), Not typable; -, no isolates in this group or category. RFLP types I, II, 46, and miscellaneous are the molecular types described in the text.

\begin{tabular}{|c|c|c|c|c|}
\hline \multirow{2}{*}{$\begin{array}{l}\text { RFLP } \\
\text { type }\end{array}$} & \multicolumn{4}{|c|}{ Epidemiological category } \\
\hline & 1 & 2 & 3 & 4 \\
\hline I & $\begin{array}{r}1(85) \\
19(\mathrm{nt})\end{array}$ & $10(\mathrm{nt})$ & $1(29 / 85 / 90)$ & $5(\mathrm{nt})$ \\
\hline II & - & $2(29 / 85 / 95 / 90)$ & $\begin{array}{l}3(29 / 85 / 95 / 90) \\
1(29 / 79 / 84 / 85 / 95 / 90)\end{array}$ & $6(29 / 85 / 95 / 90)$ \\
\hline 46 & - & $1(85 / 90)$ & $1(53 / 83 \mathrm{~A} / 85 / 95 / 90)$ & $1(85 / 90)$ \\
\hline Miscellaneous & $\begin{array}{l}1(47 / 83 \mathrm{~A} / 85 / 95 / 90) \\
1 \text { (nt) }\end{array}$ & - & $2(85)$ & $\begin{array}{l}1(53 / 77 / 85 / 90) \\
2 \text { (nt) }\end{array}$ \\
\hline
\end{tabular}


values for $p$ (the deduced genetic distance between pairs of strains) were determined. Zero genetic distance corresponds to identical fragment patterns on the gels (and thus identical location of those endonuclease sites tested throughout the genome), whereas the largest values of $p(0 \cdot 11)$ corresponded to similarities in only four fragments of the 13 and 22 fragments of the isolate pair. Pairwise comparison of $p$ for all isolates was used to calculate a dendrogram (Fig. 4) as described in Methods.

The dendrogram (Fig. 4) confirmed the qualitative analysis (Fig. 2), and showed groupings defined by values of $p$ which conform with types I, II, and 46, within each of which there are subtypes. Members of the miscellaneous group showed more disparate genetic relatedness.

\section{Relationship of genetic distance data to epidemiological and phage typing data}

Of the isolates from the intensive care units (epidemiological categories 1 and 2), 30 of 35 were RFLP type I (Table 1). Epidemiological categories 3 and 4 contained all RFLP types. RFLP type II and the miscellaneous group were more widely distributed than type I. The subtypes of types I and II were distributed as shown in the lower part of Fig. 2; subtypes I (a), (c) and (d) were concentrated in the neonatal unit, while subtype I (b) was recovered only from patients in the general intensive care unit.

When all isolates were tested with the standard international set of typing phages, typing patterns were established for only 21 of them, distributed among the molecular and epidemiological types as shown in Table 1. All strains not typable showed weak lysis with the experimental phages 88 and $87 \mathrm{M}$ (Vickery et al., 1986).

\section{Discussion}

\section{Significance of the RFLP data}

RFLP analysis has been widely used in the genetic 'fingerprinting' of eukaryotes, using probes to detect a small number of fragments (among many thousands) that carry specific sequences hybridizing with the probe; flanking sequences at highly variable loci are uniquely characteristic for each individual, and thus the number and sizes of the fragments hybridizing with the probe can be used for specific identification. In the analysis of $S$. aureus isolates described here, RFLPs were determined for the entire genome, except for those fragments so small that they would not bind sufficient ethidium bromide to be visible, or would have run off the end of the gel during electrophoresis; such fragments would be smaller than $10 \mathrm{~kb}$, and thus amount to less than $0.4 \%$ of the total genome.

Differences and similarities between staphylococcal isolates are readily visualized from the FIGE patterns, and a general grouping of the isolates can be established in this way. Others have also recently used this approach to identify differences among clinical isolates of Escherichia coli (Arbeit et al., 1990) and of S. aureus (Goering \& Duensing, 1990). However, a more quantitative specification of clones and lineages can be derived by subjecting RFLP data to the analytical procedures described by Upholt (1977) and Nei \& Li (1979).

Certain assumptions are inherent in the statistical basis of this type of analysis, the most important being that the changes which are seen in the fragment size and number result from simple point mutations which change endonuclease recognition sites. Insertions, deletions or transpositions are not theoretically accommodated in this model. However, these theoretical exclusions do not invalidate the analysis of genetic similarity, since transpositional or translocational rearrangements in general will have genetic outcomes quantitatively similar to point mutations, i.e. result in similar changes to the fragment patterns. Thus, a point mutation leading to loss or gain of a single $S m a$ I site results in a threefragment difference between parental and mutant genomes, which in a genome with $20 \mathrm{SmaI}$ sites (giving rise to 20 fragments) represents a nucleotide difference value for $p$ of 0.004 . A single insertion (or deletion) of a transposon (not containing a SmaI site), resulting in a new fragment measurably greater (or smaller) in size, would generate a two-band difference between the mutant and parent. The calculated value of $p$ from this difference would be 0.003 , i.e. slightly less than seen with a point mutation, where no change in the overall size of the chromosome results. If the inserted sequence contains a SmaI site, then the consequences would be operationally the same as those seen when a point mutation introduces a new SmaI site, i.e. a threefragment difference. Thus, a single genetic event such as a point mutation that results in a gain or loss of site, or a deletion, insertion or inversion that involves at most one site in a genome containing $20 \mathrm{SmaI}$ sites, will change the $p$ value by $0.003-0.004$. Accordingly, $p$ values can be used as a general measure of the number of events generating genetic distance.

The advantage of the analytical method used here is that it gives a single value $(p)$ to indicate essential differences and similarities, rather than attempting to describe what are otherwise qualitative differences in band patterns (position and numbers of bands on gels). It identifies those isolates which are identical or differ only to a minor degree, those which differ markedly, and those which lie somewhere in between. A greater 
measure of precision could be obtained by using a number of endonucleases, each generating a suitable number of fragments (20-30) resolvable by FIGE, or an endonuclease generating a larger number of fragments. Such additional data would provide incrementally more precise values of $p$. Thus, for a genome containing 40 endonuclease sites, a detectable point mutation in one strain (resulting in 3 fragments different, 77 fragments common) gives a $p$ value of 0.002 ; for a genome containing 80 sites, a $p$ value of 0.001 results from a single detectable point mutation. This shows the greater precision in estimates of $p$ which are potentially achievable as the fragment number increases. However, a practical limit is reached because it is often difficult to distinguish individual bands when there are more than 20-30 present in each track of FIGE gels prepared and run as described in this study. Also, changes in $p$ value of this order would not greatly change the general pattern of relationships illustrated by the dendrogram (Fig. 4).

It should also be said that when deciding whether bands on gels coincide exactly or not, the tendency in cases of uncertainty is to score bands as the same in size, rather than different. Estimates of $p$ will thus err towards the conservative, but the important point again is that the values of $p$ obtained should be seen not as precise quantitative estimates of genetic distance, but as useful summary values indicative of overall genomic similarities and differences between different isolates. So, $p$ provides an estimate of pattern similarity and thus the means to illustrate relationships within a large body of comparative data.

Scanning the data for $p$ differences between isolates indicates that the differences between isolates within each of the major RFLP types are relatively small. Thus, in type $\mathrm{I}$, the greatest difference in $p$ values is 0.011 , representing a six-fragment difference between subtypes (a) and ( $\left.c^{\prime \prime}\right)$, or, at the molecular level, two or three events of the sort described above. A number of the subtypes within both RFLP types I and II differ from each other apparently by the equivalent of a single detectable genetic event.

BssHII generates more fragments than SmaI, and resolution of fragments accordingly is more difficult; the subdivision of types is thus less clear with this enzyme. However, the results obtained with BssHII show the same division into major types, in accord with those obtained with $S m a I$, and providing further validation of the method.

\section{Correlation of epidemiological and molecular data}

The results suggest that two major lineages of MRSA have spread through the two hospitals, with miscellan- eous types, presumably of diverse origin, interspersed among them. Significantly, 30 of the 35 isolates found in epidemiological categories 1 and 2 ( $\mathrm{RCH}$ intensive care units) are RFLP type I, suggesting that a single clonal type became established in these units, and that this clone subsequently evolved into a lineage of several subclones.

Two of the three isolates of RFLP type 46 were detected in patients who were not infected in Canberra hospitals. This RFLP type is identical to the strain ANS46, first isolated in a Melbourne hospital in 1982. Thus, an MRSA strain prevalent in Melbourne about seven years ago is currently present in the Canberra hospitals and an outlying rural hospital.

Though RFLP type II isolates form a coherent molecular group, they come from diverse epidemiological sources. This suggests that RFLP type II is more generally distributed, and might accordingly be part of an earlier epidemic in the Canberra hospitals than that represented by the type I clone.

The miscellaneous molecular group, not surprisingly, was obtained from patients in all locations sampled, and presumably represents part of the background of MRSA infections which occur currently in most major hospitals in eastern Australia (Turnidge et al., 1989).

Phage typing results correlated with RFLP analysis for type II and type 46 isolates, while the miscellaneous RFLP types exhibited a range of susceptibilities to the standard phage set. Phage typing also identified the type I isolates as a group through their non-typability by the standard set. In each case, RFLP analysis provided further detail within RFLP groups by subdividing them into subtypes.

Analysis of RFLPs by FIGE provides a means by which groups of isolates and strains may be compared at the genetic level. Importantly, it may reveal the extent of genetic change which has occurred or is occurring in staphylococcal populations. To this extent, it could provide an alternative approach to identifying the nature of the determinants of virulence is staphylococci, and in particular, those genes and combinations of genes found in highly virulent epidemic strains but not in avirulent types. The persistent presence of particular genes or gene combinations in epidemic strains may reveal genetic patterns which, by reference to the data now accumulating as a genetic map of $S$. aureus (Pattee, 1990), will provide a more precise assessment of the elements determining the virulence and thus the pathological impact of different strains of this important clinical opportunist.

This work was supported by the National Health and Medical Research Council of Australia. We thank Libby Viccars for excellent technical assistance. 


\section{References}

Arbeit, R. D., Arthur, M., Dunn, R., Kim, C., Selander, R. K. \& GoLDSTEIN, R. (1990). Resolution of recent evolutionary divergence among Escherichia coli from related lineages: the application of pulsed field electrophoresis to molecular epidemiology. Journal of Infectious Diseases 161, 230-235.

Berke, A. \& Tilton, R. C. (1986). Evaluation of rapid coagulase methods for the identification of Staphylococcus aureus. Journal of Clinical Microbiology 23, 916-919.

Blair, J. E. \& Williams, R. E. (1961). Phage typing of staphylococci. Bulletin of the World Health Organization 24, 771-784.

Carle, G. F., Frank, H. \& Olson, M. H. (1986). Electrophoretic separations of large DNA molecules by periodic inversion of the electric field. Science 232, 65-68.

Coia, J. E., Thomson-Carter, F., Baird, D. \& Platt, D. J. (1990). Characterisation of methicillin-resistant Staphylococcus aureus by biotyping, immunoblotting and restriction enzyme fragmentation patterns. Journal of Medical Microbiology 31, 125-132.

CoOkson, B., Talsania, H., Naidoo, J. \& Phillips, I. (1986). Strategies for typing and properties of epidemic methicillin resistant Staphylococcus aureus. European Journal of Clinical Microbiology 5, 702-709.

Goering, R. V. \& Duensing, T. D. (1990). Rapid field-inversion gel electrophoresis in combination with a rRNA gene probe in the epidemiological evaluation of staphylococci. Journal of Clinical Microbiology 28, 426-429.

Heineine, N. \& Stewart, P. R. (1986). Physiological determination of methicillin resistance in Staphylococcus aureus: comparison of clinical and genetically derived isolates. Journal of Antimicrobial Chemotherapy 17, 705-715.

Inglis, B., Matthews, P. R. \& Stewart, P. R. (1990). Induced deletions within a cluster of resistance genes in the mec region of the chromosome of Staphylococcus aureus. Journal of General Microbiology 136, 2231-2239.

LyoN, B. R., MAY, J. W. \& Skurray, R. A. (1983). Analysis of plasmids in nosocomial strains of multiple antibiotic-resistant Staphylococcus aureus. Antimicrobial Agents and Chemotherapy 23, 817-826.

Matthews, P. R., ReEd, K. C. \& Stewart, P. R. (1987). The cloning of chromosomal DNA associated with methicillin and other resistances in Staphylococcus aureus. Journal of General Microbiology 133, 1919-1929.
Mulligan, M. E., Kwok, R. Y., Citron, D. M., John, J. F. \& Smith, P. B. (1988). Immunoblots, antimicrobial resistance, and bacteriophage typing of oxacillin-resistant Staphylococcus aureus. Journal of Clinical Microbiology 26, 2395-2401.

National Committer for Clinical Laboratory Standards (1986). Approved standard methods for dilution antimicrobial susceptibility tests for bacteria that grow aerobically. In Laboratory Standard 5, pp. 579-615. Villanova, PA: National Committee for Clinical Laboratory Standards.

NEI, M. \& LI, W. H. (1979). Mathematical model for studying genetic variation in terms of restriction endonucleases. Proceedings of the National Academy of Sciences of the United States of America 76, 5269-5273.

Pattee, P. A. (1990). Staphylococcus aureus. Genetic Maps 5, 22-27.

SaIToU, N. \& NEI, M. (1987). The neighbour-joining method: a new method for reconstructing phylogenetic trees. Molecular Biology and Evolution 4, 406-425.

SCHLEIFER, K. H. \& FISHER, U. (1982). Description of a new species of the genus Staphylococcus: Staphylococcus carnosus. International Journal of Systematic Bacteriology 32, 153-156.

Skurray, R. A., Rouch, D. A., Lyon, B. R., Gillespie, M. T., Tennent, J. M., Byrne, M. E., Messerotti, L. J. \& May, J. W. (1988). Multiresistant Staphylococcus aureus: genetics and evolution of epidemic Australian strains. Journal of Antimicrobial Chemotherapy 21 (Suppl. C), 19-38.

SMITH, S. M. \& BerezNy, C. (1986). Comparative evaluation of identification systems for testing methicillin-resistant strains of Staphylococcus aureus. Journal of Clinical Microbiology 24, 173176

Smith, C. L., Warburton, P. E., Gaal, A. \& Cantor, C. R. (1986) Analysis of genome organization and rearrangements by pulsefield gradient gel electrophoresis. Genetic Engineering 8, 45-70.

Turnidge, J., LAWson, P., Munro, R. \& BenN, R. (1989). A national survey of antimicrobial resistance in Staphylococcus aureus in Australian teaching hospitals. Medical Journal of Australia 150, 6572.

UPHOLT, W. B. (1977). Estimation of DNA sequence divergence from comparison of restriction endonuclease digests. Nucleic Acids Research 4, 1257-1265.

Vickery, A. M., Beard-Pegler, M. A. \& Stubbs, E. (1986). Phagetyping patterns and lysogenicity of methicillin-resistant strains of Staphylococcus aureus from Sydney, Australia, 1965-1985. Journal of Medical Microbiology 22, 209-216. 